\title{
An inability of $;$-acetylenic GABA to block eating evoked by hypothalamic stimulation
}

\author{
ELLIOT S. VALENSTEIN and MICHAEL S. MYSLOBODSKY*
}

Dept. of Psychology and Neuroscience, University of Michigan, Ann Arbor, Mich. 48109 (U.S.A.)

(Received April 4th, 1980)

(Accepted April 7th, 1980)

Key words: hypothalamic stimulation - stimulation-evoked eating - GABA-transaminase inhibitors - hypersynchronous EEG

Several lines of evidence have implicated the nigrostriatal dopamine system and the neocortex for eating occurring either spontaneously or in response to electrical stimulation of the lateral hypothalamus (ESLH). GABA-ergic neural systems are known to modulate activity in the nigrostriatal system and neocortex. The GABA-transaminase inhibitor, $\gamma$-acetylenic GABA (GAG) was administered to rats eating in response to ESLH. This drug produced hypersynchronization of the cortical EEG and behavioral sedation, but did not alter the current threshold for evoking eating by ESLH. Implications of results for understanding mechanisms underlying ESLH-induced eating are discussed.

Although it has been known for over 20 years that hypothalamic stimulation may evoke eating in satiated animals [10], the mechanisms underlying this fascinating phenomenon remain unclear. A number of investigators have argued that the eating observed during electrical stimulation of the lateral hypothalamus (ESLH) results from activation of specific neural circuits regulating hunger $[7,16]$. In contrast, Valenstein and his colleagues have demonstrated that the eating and other behaviors evoked by ESLH are much more stereotyped, and differ in other significant ways from the eating of food-deprived animals [26-28]. Moreovir, Cox and Valenstein [6] have shown that the neural substrate for this behavior was not as specific as implied by investigators committed to the idea of congruence with the feeding area as defined by 'lesion studies.' Recently, evidence that the behavior evoked by ESLH persists after destruction of hypothalamic tissue surrounding the tip of the stimulating electrode provided additional support for the view that such behavior does not depend on activation of specific neural circuits [3]. The demonstration that such non-specific stimu-

- Permanent address: Psychobiology Research Unit, Dept. of Psychology, Tel-Aviv University, Tel-Aviv, Israel. 
lation as tail-pinch can evoke eating in satiated animals has also made it more difficult to maintain that such behavior reflects the activation of specific neural substrate of hunger $[1,2]$.

Several lines of evidence suggest that neural circuits outside the so-called 'classical lateral hypothalamic feeding area' may contribute significantly to the capacity of ESLH to evoke eating. Following Ungerstedt's [25] demonstration that disruption of the nigrostriatal dopamine system prodices severe feeding deficits, impairment of stimulation-induced feeding was reported after intraventricular 6-hydroxydopamine (6-OHDA) administration [18] and after intraperitoneal injections of the dopamine receptor blocker, haloperidol [19]. Antelman et al. [2] have also provided evidence for an important role of dopamine in tail-pinch-evoked eating. Additional support for the importance of the nigrostriatal systems derives from the evidence from both lesion and electrical stimulation studies which demonstrate the importance of the corpus striatum for sensory-motor reflexes involved in feeding behavior [13, 14, 17, 22, 24].

It is generally accepted that activity in the nigrostriatal system is modulated by an inhibitory, striatonigral GABAergic feedback system originating in the globus pallidus and caudate nucleus and terminating in the substantia nigra $[9,20,32]$. An inhibitory role for GABA in feeding behavior was suggested by Kuriyama and Kimura [12], who reported that GABA levels were decreased in the lateral hypothalamus, but increased in the ventromedial hypothalamus, by insulin-induced hypoglycemia. Moreover, although Soper and Wise [21] attributed the facilitation of ESLH-induced eating by diazepam to the antianxiety action of this drug, several investigators have suggested that diazepam action is mediated by its effect on GABAergic activity (e.g. refs. 5 and 23). The purpose of the present study, therefore, was to assess the effects of modifying GABA brain levels on eating induced by ESLH.

The experimental animals were male rats (body weight ranged from 285 to $600 \mathrm{~g}$ ) of either the Long-Evans (Charles River laboratories, Wilmington, Mass. and the Simonsen Co., Gilroy, Calif.) or Sprague-Dawley strains (Holtzman Laboratories, Madison, Wisc.). Animals were housed in a temperature-regulated vivarium in which the lights were off from 12.00 to $22.00 \mathrm{~h}$. Daily body weight records were maintained on all animals.

Animals were anesthetized with Equi-Thesin (Jen-Sal, Inc.) supplemented, when r.eeded, by Metofane (methoxyflurane, Pitman-Moore, Inc.). Bipolar electrodes were implanted in the lateral hypothalamus using standard stereotaxic techniques (coordinates: $3.5 \mathrm{~mm}$ posterior to bregma, $1.4 \mathrm{~mm}$ lateral, $8.25 \mathrm{~mm}$ below the surface of the skull level between bregma and lambda). The electrodes were twisted, bipolar stainless steel wires $(0.25 \mathrm{~mm}$ diameter, MS 303/1, Plastic Products Co., Roanoke, Va.) bare of insulation only at the adjacent tips.

To modify GABA brain activity the newly synthesized GABA-trans- 
aminase inhibitor, $\gamma$-acetylenic GABA (GAG) was used. The drug was administered by intraperitoneal injections. GAG was injected $4 \mathrm{~h}$ prior to all behavioral or electrophysiological testing. Dose levels were $100 \mathrm{mg} / \mathrm{kg}$ body wt. on the first drug-test day followed by $50 \mathrm{mg} / \mathrm{kg}$ body wt. on each of 5 consecutive days. This dose level has been shown to produce a 6-fold increase in the brain GABA content, the effect being maximal $4 \mathrm{~h}$ after treatment, remaining almost on the same level $12 \mathrm{~h}$ later and declining to slightly above normal levels by $24 \mathrm{~h}$ after treatment $[11,29]$.

Because of the report that GAG produces somnolence and sedation accompanied by synchronous and hypersynchronous (wave-spike) electrographic activity, additional animals were implanted with bilateral cortical, recording electrodes in order to demonstrate the effectiveness of our drug regimen. Holes for electrodes, located over the visual cortex, were drilled in the midline over the cerebellum. The cortical and indifferent electrodes consisted of approximately $1.0 \mathrm{~mm}$ silver balls (produced by melting insulated silver wire, $0.1 \mathrm{~mm}$ diameter) positioned on the dura. The wire was presoldered to Amphenol microminiature pins, which were centered on the skull. One week after surgery, these animals received GAG for 4 consecutive days according to the protocol described above. EEG was monitored 4-5 $\mathrm{h}$ after injection - a time period corresponding to that of all behavioral testing. The EEG record clearly demonstrated a slowing (3-4 Hz) and hypersynchronization with paroxysmal waves of wave-spike discharge. This pattern developed after the first GAG injection and intensified following successive injections.

Following recovery from surgery, rats with hypothalamic electrodes were placed in a $20.5 \times 26.5 \times 42.5 \mathrm{~cm}$ Plexiglas chamber with Noyes $45 \mathrm{mg}$ food pellets scattered on the floor. After a $20 \mathrm{~min}$ habituation period animals received $20 \mathrm{sec}$ trains of hypothalamic stimulation (60 Hz sine waves) from a constant current source, alternating with $15 \mathrm{sec}$ inter-trial intervals. Rats that consistently ate food pellets during ESLH were then placed on a regimen of 'threshold testing.' The 'threshold test' is a measure of the minimal stimulation intensity that evoked eating. It consisted of a 'staircase titration' method in which the intensity was raised in $1 \mu \mathrm{A}$ steps if no eating was observed, but was repeated at the same intensity when eating occurred. If eating did not recur, the intensity was raised again. When eating recurred at the same intensity, the current was decreased $3 \mu \mathrm{A}$, and the procedure repeated twice more. The three intensities at which eating occurred twice in succession were averaged for the 'daily threshold.'

Ovar the 6 days of GAG injections, all 5 rats exhibited a significant decrease in body weight. On the last day of GAG treatment the animals were at $80 \%$ of the body weight they would have reached had they not received this drug. During the first 4 days of GAG injections the muscle tone of the animals 
was obviously flaccid; they tended to hang limply when picked up and they showed little spontaneous behavior during testing. During the last 2 days some spontaneity and muscle tone appeared to return.

In spite of the very significant decrease in body weight during the 6 days of GAG treatment, in addition to the behavioral signs of sedation, the threshold of ESLH-evoked eating showed only a relatively small (non-significant) increase. When compared to the average current threshold for the 3 days following drug treatment no difference at all was evident. It may be worth noting that the variability of the thresholds was greater during the post-drug tests. The increased variability may have been the result of individual differences in 'rebound effect' following GAG treatment. We have observed that some, but not all, animals become very jumpy and may even attempt to bite when handled after the termination of several days of GAG treatment. Fig. 1 depicts the results of the daily threshold tests prior to, during, and after GAG treatment.

Even though there was no significant change in threshold during GAG treatment, there were qualitative differences in the behavior of animals during hypothalamic stimulation. Prior to GAG treatment, the rats tended to be quite active and aroused during stimulation and often ate a number of pellets during the $20 \mathrm{sec}$ period. In contrast, under the influence of GAG treatment, the animals often sat quietly until the last $5 \mathrm{sec}$ of stimulation, at which time they

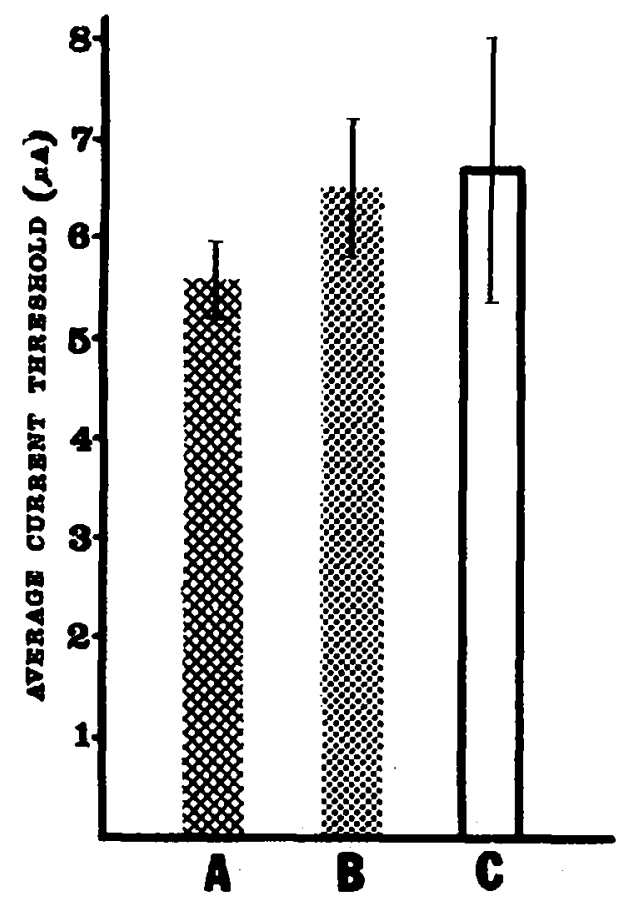

Fig. 1. Average current threshold ( \pm 5.E.M.) for evoking eating fr.'m stimulation of lateral hypothalamic stimulation of rats $(n=5)$ beiore $(A)$, during $(B)$ and after $(C) 6$ days of treatment with ;-actstylenic GABA (GAG). See text for schedule of drug dose administration. 
sometimes moved only their head and ate a single pellet. On a numbe: of occasions, the GAG-treated animal was not activated by the ESLH until only 1 or $2 \mathrm{sec}$ of stimulations remained, leaving insufficient time for the animal to do more than move its head toward the pellet. In most such instances, the following stimulation (being $1 \mu \mathrm{A}$ higher) activated the animal more quickly and one or more pellets were eaten. Thus, the major effect of the GAG treatment seemed to be on the capacity of the stimulation to arouse a sedated animal. Once aroused, the animals appeared to be motivated to eat.

The present results demonstrate that the GABA-transaminase inhibitor, GAG, does not significantly affect the current threshold for evoking eating by ESLH. These results are surprising in view of the evidence that GABA plays a critical role in modulating the dopamine pathways connecting the substantia nigra and the corpus striatum, a neural circuitry implicated in feeding mechanisms by the several lines of evidence discussed above. Under the influence of GAG, our animals not only failed to engage in exploratory behavior when placed in the test chamber, but they also did not respond to such noxious stimulation as hard tail-pinching. The significant weight loss experienced by all animals receiving GAG may also have been due to a lack of responsiveness to the food available in their home cages. The present finding that the threshold for evoking eating behavior during ESLH is relatively unchanged suggests either that such behavior does not depend upon responsiveness to external stimuli, or that the hypothalamic stimulation is capable of potentiating responsiveness to stimuli. The latter possibility is consistent with observations and views of several investigators $[4,8,15,30,31]$. In any case, the major effect of GAG treatment on ESLH-evoked eating appears to be on sedation rather than on motivation to eat.

\section{ACKNOWLEDGEMENTS}

The authors are pleased to acknowledge the generous gift of $\gamma$-acetylenic GABA by the Merrell National Laboratories, Cincinnati, Ohio.

\section{REFERENCES}

1 Antelman. S.M. and Szechtman, H.. Tail pinch induces eating in sated rats which appears to depend on nigrostriatal dopamine, Science, 189 (1975) 731-733.

2 Antelman, S.M., Szechtman, H., Chin, P. and Fisher, A.E., Tail pinch-induced eating, gnawing and licking behavior in rats: dependence on the nigrostriatal dopamine system, Brain Res., 99 (1975) 319-337.

3 Bachus, S.E. and Valenstein, E.S., Individual behavioral responses to hypothalamic stimulation persist despite destruction of tissue surrounding electrode tip, Physiol. Behav., 23 (1979) 421-426.

4 Beagley, W.K. and Holley, T.L., Hypothalamic stimulation facilitates contralateral visual control of a learned response, Science, 196 (1977) 321-322. 
5 Bloom, F.E., Neural mechanisms of benzodiazepine actions, Amer. J. Psychiat., 134 (1977) 669-672.

6 Cox, V.C. and Valenstein. E.S., Distribution of hypothalamic sites yielding stimulus-bound behavior, Brain Behav. Evol., 2 (1969) 359-376.

7 Devor, M.G., Wise, R.A., Milgram, N.W. and Hoebel. B.G., Physiological control of hypothalamically elicited feeding and drinking, J. comp. physiol. Psychol., 73 (1970) 226-232.

8 Flynn, J.P., Edwards, S.B. and Bandler, R.J., Changes in sensory and motor systems during centrally elicited attack, Behav. Sci., 16 (1971) 1-19.

9 Grofova, I. and Rinvik, E., An experimental electron microscopic study on the striatonigral projection in the cat, Exp. Brain Res., 11 (1970) 249-262.

10 Hess, W.R., The Functional Organization of the Diencephalon, Grune and Stratton, New York, 1957.

11 Jung, M.J., Lippert, B., Metcalf, B.W.. Schechter, P.J., Bohlen, P. and Sjoerdsma, A., The effect of 4-aminohex-5-ynoic acid (;-acetylenic GABA,;-ethynyl GABA), a catalytic inhibitor of GABA transaminase, on brain GABA metabolism in vivo, J. Neurochem., 28 (1977) 717-723.

12 Kuriyama, K. and Kimura, H., Distribution and possible functional roles of GABA in the retina, lower auditory pathway, and hypothalamus. In E. Roberts, T.N. Chase and D.B. Tower (Eds.), GABA in Nervous System Function, Raven Press, New: York, 1976, pp. 203-216.

13 Levine, M.S., Ferguson, N., Kreinick, C.J., Gustafson, J.W. and Schwartzbaum, J.S., Sensorimotor dysfunctions and aphagia and adipsia following pallidal lesions in rats, J. comp. physiol. Psycho!., 77 (1971) 282-293.

14 Levine, M.S. and Schwartzbaum, J.S., Sensori-motor functions of the striatopallidal system and lateral hypothalamus and consummatory behavior in rats, J. comp. physiol. Psychol., 85 (1973) 615-635.

15 Marshall, J.F. and Teitelbaum, P., New considerations in the neuropsychology of motivated behaviors. In L.L. Iversen, S.D. Iversen and S.H. Snyder (Eds.), Handbook of Psychopharmacology, Vol. 7, Plenum, New York, 1977, pp. 201-229.

16 Miller, N.E., Motivational effects of brain stimulation and drugs, Fed. Proc., 19 (1960) 846-854.

17 Morgane, P.J., Alterations in feeding and drinking behavior of rats with lesions in globi pallidi, Amer. J. Physiol., 201 (1961) 420-428.

18 Phillips, A.G. and Fibiger, H.C.. Deficits in stimulation-induced feeding after intraventricular administration of 6-hydroxydopamine in rats, Behav. Biol., 9 (1973) 749-754.

19 Phillips, A.G. and Nikaido, R.S., Disruption of brain stimulation-induced feeding by dopamine receptor blockade, Nature (Lond.), 258 (1975) 750-751.

20 Rinvik, E. and Grofova, I., Observations on the fine structure of the substantia nigra in the cat, Exp. Brain Res., 11 (1970) 229-248.

21 Soper, W.Y. and Wise, R.A., Hypothalamically induced eating; eating from 'non-eaters' with diazepam, T.I.T. J. Life Sci., 1 (1971) 79-84.

22 Sorenson, C.A. and Ellison, G.D., Striatal organization of feeding behavior in the decorticate rat, Exp. Neurol., 29 (1970) 162-174.

23 Stein, L., Belluzzi, J.D. and Wise, C.D., Benzodiazepines: behavioral and neurochemical mechanisms, Amer. J. Psychiat., 134 (1977) 665-669.

24 Szabo, I., Sarkisian, J.S., Lenard, L. and Nemeth, L., Pallidal stimulation in rats: Facilitation of stimulation-induced chewing by food and water deprivation, Physiol. Behav., 18 (1977) 361-368.

25 Ungerstedt, U., Stereotaxic mapping of the monoamine pathways in the rat brain, Acta physiol. scand., 376, Suppl. (1971) 1-48. 
26 Valenstein, E.S., Brain mechanisms of reinforcement. In W.H. Sweet, S. Obrador and J. Martin-Rodriguez (Eds.), Neurosurgical Treatment in Psychiatry, Pain and Epilepsy, University Park Press, Maryland, 1977, pp. 27-49.

27 Valenstein, E.S., Brain stimulation and behavior control. In J.K. Cole and T.B. Sonderegger (Eds.), Nebraska Symposium on Motivation, 1974, University of Nebraska Press, Nebraska, 1975, pp. 251-292.

28 Valenstein, E.S., Cox, V.C. and Kakolewski, J.W., Reexamination of the role of the hypothalamus in motivation, Psychol. Rev., 77 (1970) 16-31.

29 Wood, J.D., Durham, J.S. and Peesker, S.J., Effect of di-n-propylacetate and $;$-acetylenic GABA on hyperbaric oxygen-induced seizures and GABA metabelism, Neurochem. Res., 2 (1977) 707-715.

30 Wright, J.J. and Craggs, M.D., Arousal and intracranial self-stimulation in split-brain monkeys, Exp. Neurol., 55 (1977) 295-303.

31 Wright, J.J. and Craggs, M.D., Intracranial self-stimulation, cortical arousal, and the sensorimotor neglect syndrome, Exp. Neurol., 65 (1979) 42-52.

32 Yoshida, M. and Precht, W., Monosynaptic inhibition of neurons of the substantia nigra by caudatonigral fibers, Brain Res., 32 (1971) 225-228. 